\title{
Statistical and RBF NN models: Providing forecasts and risk assessment
}

\author{
Milan MARČEK, University of Žilina ${ }^{\mathrm{i}, \mathrm{ii}}$
}

\begin{abstract}
Forecast accuracy of economic and financial processes is a popular measure for quantifying the risk in decision making. In this paper, we develop forecasting models based on statistical (stochastic) methods, sometimes called hard computing, and on a soft method using granular computing. We consider the accuracy of forecasting models as a measure for risk evaluation. It is found that the risk estimation process based on soft methods is simplified and less critical to the question whether the data is true crisp or white noise.
\end{abstract}

\section{Keywords}

ARCH-GARCH models, forecasting accuracy, granular computing, soft neural networks, time series.

JEL Classification: C10, C53, D81

\footnotetext{
${ }^{\mathrm{i}}$ Faculty of Management Science and Informatics, University of Zilina, Univerzitná 8215/1, 01026 Žilina.

${ }^{\text {ii }}$ Messer Tatragas, spol. s r. o., Chalupkova 9, 81944 Bratislava, Slovak Republic. milan.marcek@messergroup.com (corresponding email)

This work was supported by grant foundation from the Grant Agency of the Czech Republic under the grant No. 402/08/0022.
}

\section{Introduction}

The evaluation of risk is a key element of management decision making. The ultimate effectiveness of any decision depends on the ability to predict the uncontrollable aspects of events following the decision. The purpose of forecasting is to reduce the risk in decision making. The concept of forecast accuracy has been adopted as a measure for quantifying the risk relatively recently. By devoting more resource to forecasting, we should be able to improve our forecasting accuracy and thereby eliminate some of the losses resulting from uncertainty in the decision-making process.

The term risk has numerous different meanings. To emphasize the negative aspects of risk we refer to the definition of Huang and Raun (2008): Risk is a scene in the future associated with some adverse incident. Based on knowledge and information about risks, the risk is classified into five categories (as in Huang, 2007): pseudo risk, probability risk, fuzzy risk, uncertain risk, and fuzzy-stochastic risk. Fuzzy and uncertain risk is modeled by fuzzy methodology where input data are not determined precisely but only softly. For example these methodologies are used for the concept of the value at risk for the measuring of their trading portfolios. This approach uses fuzzy numbers (see e.g. Yoshida (2003), Zmeškal (2005). Probability risk is defined as a scene in the future with a specific adverse incident that we are able to statistically predict by using probability models and a lot of data. Probability risk, in contrast with fuzzy measures, can be assessed in terms of probability. Input data and model's parameters are determined as real numbers. These assumptions are fulfilled in many economic and financial situations by risk measuring, e.g. in time series modeling and forecasting and VaR methods Marček (2009), Zmeškal (2005)), methods based on the extreme value theory (Havlický (2009), Lévy models (Tichý (2006), Applebaum (2004)) and so on.

Machine (artificial) intelligence systems as neural networks are dynamical trainable adaptive systems defined with many arbitrarily interacted differential or 
difference equations. Unlike statistical estimators they learn from experience with numerical sample data. Dynamical systems may or may not by stable. Their state variables change over time. Any change of the system consumes energy. In Kosko (1992) the stability of neural networks has been proven by global Lyapunov energy functions. One of most methods for finding the optimal weights of dynamical systems, i.e. a minimum of energy functions, is the gradient method. For more details about energy equations of generalized mechanical systems see Han and Zhang (2001).

Forecasting systems are usually a part of larger management systems and, as a subsystem, interacts with other components of the total system to determine overall performance. Ideally the forecasting system should provide a description of forecast error as well as a forecast. The forecasting process should result in an estimate of the probability distribution of the variable being predicted. This permits risk to be objectively incorporated into the decision-making process. If the forecasting system yields unbiased estimates, then the traditional statistical summary measure of forecast accuracy used by academicians and practitioners is expected the Root Mean Squared Error (RMSE) that is equal to the standard forecast error. Another measure of the uncertainty of forecasts is the coefficient of variations (CV) defined as RMSE $/ \bar{y}$ which expresses the unexplained variability remaining in the data relative to the average value $\bar{y}$ of the modeled variable. If the CV is less than 0.05 , then we have a fairly good model. It is seen that the risk value of the predicted variable is proportional to the accuracy of forecasting models.

This article develops nonlinear forecasting models, analyses, discusses and compares the forecast accuracy from models which are derived from competing statistical and Radial Basic Function (RBF) neural network (NN) specifications. Our motivation for this comparative study lies in both the difficulty for constructing of appropriate statistical Autoregressive/Generalised Conditionally Heteroscedastic (ARCH-GARCH) models (so called hard computing) to forecast volatility even in ex post simulations and the recently emerging problemsolving methods that exploit tolerance for impression to achieve low solution costs (soft computing). The aim of the paper is to explain, and to show the achieved aspects of both the statistical and soft approach for quantifying forecast accuracy applied to daily VUB bond price time series which is, according to the definition by Zivot and Wang (2005), the high frequency data sequence.

The paper is organized in the following manner. In Section 2 we briefly describe the basic methodology of ARCH-GARCH models. In Section 3 we present the data, conduct some preliminary analysis of the time series and demonstrate the forecasting abilities of ARCH-GARCH modes of an application. Section 4 is devoted to a granular computing approach applied for RBF neural networks. This approach is suggested as an alternative to the approach analyzed in Section 3. In Section 5 we put an empirical comparison. Section 6 briefly concludes.

\section{Some ARCH-GARCH models for financial data}

ARCH models are considered as some specific nonlinear time series models, which allows for quite an exhaustive study of the underlying dynamics. ARCHGARCH models are designed to capture certain characteristics that are commonly associated with financial time series. They are among others: fat tails, volatility clustering, persistence, mean-reversion and leverage effect. As far as fat tails, it is well known that the distribution of many high frequency financial time series usually have fatter tails than a normal distribution. The phenomenon of fatter tails is also called excess kurtosis. In addition, financial time series usually exhibits a characteristics known as volatility clustering in which large changes tend to follow large changes, and small changes tend to follow small changes. Volatility is often persistent, or has a long memory if the current level of volatility affects the future level for more time periods ahead. Although financial time series can exhibit excessive volatility from time to time, volatility will eventually settle down to a long run level. The leverage effect expresses the asymmetric impact of positive and negative changes in financial time series. It means that the negative shocks in price influence the volatility differently than positive shocks of the same size. This effect appears as a form of negative correlation between the changes in prices and the changes in volatility.

The first model that provides a systematic framework for volatility modeling is the ARCH model introduced by Engle (1982). Bollerslev (1986) proposes a useful extension of Engle's ARCH model known as the generalized ARCH (GARCH) model for time sequence $\left\{y_{t}\right\}$ in the following form

$$
\begin{gathered}
y_{t}=\varepsilon_{t} \sqrt{h_{t}}, \\
h_{t}=\alpha_{0}+\sum_{i=1}^{m} \alpha_{i} y_{t-i}^{2}+\sum_{j=1}^{s} \beta_{j} h_{t-j},
\end{gathered}
$$

where $\left\{\varepsilon_{t}\right\}$ is a sequence of iid random variables with zero mean and unit variance, $\alpha_{i}$ and $\beta_{i}$ are the ARCH and GARCH parameters, $h_{t}$ represent the conditional 
variance of time series conditional on all the information to time $t-1$.

In the literature several variants of basic ARCHGARCH model (1) has been derived. If only the squared residuals $\varepsilon_{t-i}$ enter into the equation, the signs of the residuals or shocks have no effects on conditional volatility. However, a stylized fact of financial volatility is that bad news (negative shocks) tends to have a larger impact on volatility than good news (positive shocks). Nelson (1991) proposed the exponential GARCH model abbreviated as EGARCH to allow for leverage effects in the form

$$
\log h_{t}=\alpha_{0}+\sum_{i=1}^{p} \alpha_{i} \frac{\left|\hat{\varepsilon}_{t-i}\right|+\gamma_{i} \hat{\varepsilon}_{t-i}}{\sigma_{t-i}}+\sum_{j=1}^{q} \beta_{j} h_{t-j},
$$

where $\hat{\varepsilon}_{t-i}$ are residuals, $\gamma_{i}$ denotes the coefficient of leverage effects. Note if $\hat{\varepsilon}_{t-i}$ is positive or there is good news, the total effect of $\hat{\varepsilon}_{t-i}$ is $\left(1+\gamma_{i}\right) \hat{\varepsilon}_{t-i}$. However contrary to the good news, if $\hat{\varepsilon}_{t-i}$ is negative or there is bad news, the total effect of $\hat{\varepsilon}_{t-i}$ is $\left(1-\gamma_{i}\right)\left|\hat{\varepsilon}_{t-i}\right|$. Bad news can have a larger impact on the volatility. Then the value of $\gamma_{i}$ would be expected to be negative (see Zivot and Wang (2005)).

The basic GARCH model can be extended to allow for leverage effects. This is performed by treating the basic GARCH model as a special case of the power GARCH (PGARCH) model proposed by Ding et al. (1993), Granger and Engle (see also Zivot and Wang (2005)):

$$
h_{t}^{d}=\alpha_{0}+\sum_{i=1}^{p} \alpha_{i}\left(\left|\hat{\varepsilon}_{t-i}\right|+\gamma_{i} \hat{\varepsilon}_{t-i}\right)^{d}+\sum_{j=1}^{q} \beta_{j} h_{t-j}^{d}
$$

where $d$ is a positive exponent (see Zivot and Wang, 2005). Another ARCH-GARCH models as the ARCH-GARCH regression and ARCH-GARCH mean model can be found in Marček (2009, 105-130).

\section{An Application of ARCH-GARCH Models}

We illustrate the ARCH-GARCH methodology on the developing of the forecast model. The data is taken from the commercial VUB bank of the Slovak Republic. ${ }^{1}$ The data consist of daily observations for the price time series of the bond fund of VUB (BPSVUB). The data was collected for the period May 7, 2004 to February 28, 2008 which provided 954 observations (see Figure 1). All the data are expressed in natural logarithms. To build a forecast model the

1 The data are available at http://www.vubam.sk/ Default.aspx?CatID=40\&fundId $=4$. sample period $r_{1}, \ldots, r_{900}$ (training data set) for analysis was defined, and the ex post forecast period $r_{901}, \ldots, r_{954}$ (validation data set). By only using the actual and forecast values within the ex post forecasting period only, the accuracy of the model can be calculated.

Input selection is of crucial importance to the successful development of an ARCH-GARCH model. Potential inputs were chosen based on traditional statistical analysis: these included the raw BPSVUB and lags thereof. The relevant lag structure of potential inputs was analyzed using traditional statistical tools, i.e. using the autocorrelation function (ACF), partial autocorrelation function (PCF) and the Akaike/ Bayesian information criterion (AIC/BIC). We looked to determine the maximum lag for which the PACF coefficient was statistically significant and the lag given the minimum AIC. According to these criterions the ARMA(5) model was specified as

$$
r_{t}=\xi+\phi_{1} r_{t-1}+\phi_{2} r_{t-2}+\phi_{3} r_{t-3}+\phi_{4} r_{t-4}+\phi_{5} r_{t-5}+\varepsilon_{t}
$$

where $\xi, \phi_{1}, \phi_{2}, \ldots, \phi_{5}$ are unknown parameters of the model, $\varepsilon_{t}$ is independent random variable drawn from stable probability distribution with mean zero and variance $\sigma_{\varepsilon}^{2}$. The maximum likelihood procedure was used for estimation of the parameters of an ARCH or GARCH model. The quantification of the model was performed by means of R2.6. $0^{2}$ and resulted in the following mean equation:

$$
\begin{aligned}
r_{t}= & 0,0000748+0,06628 r_{t-1}+0,09557 r_{t-2}+0,0275 r_{t-3}+ \\
& 0,0528 r_{t-4}+0,09795 r_{t-5}+e_{t},
\end{aligned}
$$

and variance equation

$$
h_{t}=1,958 \cdot 10^{-8}+0,1887 e_{t-1}^{2}+0,8075 h_{t-1},
$$

where $e_{t}$ are residuals of estimated error term $\varepsilon_{t}$ from (4). The fitted vs. actual values are graphically displayed by means of Eviews ${ }^{3}$ in Figure 1.

The significance of the estimated parameters of the model was performed using the LM test. The BDS method of testing for idd was used. All the parameters of the model (6) are significant at 5\% level of significance. Identically, the BDS test performed on standardized residuals showed no evidence of nonlinearity in standardized residuals of $r_{t}$. An interested reader should refer to Brock et al. (1996) for the theoretical background of these tests.

\footnotetext{
2 http://cran.r-project.org.

${ }^{3}$ http://www.eviews.com.
} 
Next, for catching the leverage effect, the model ARMA(5,0)+EGARCH(1,1) was estimated. The

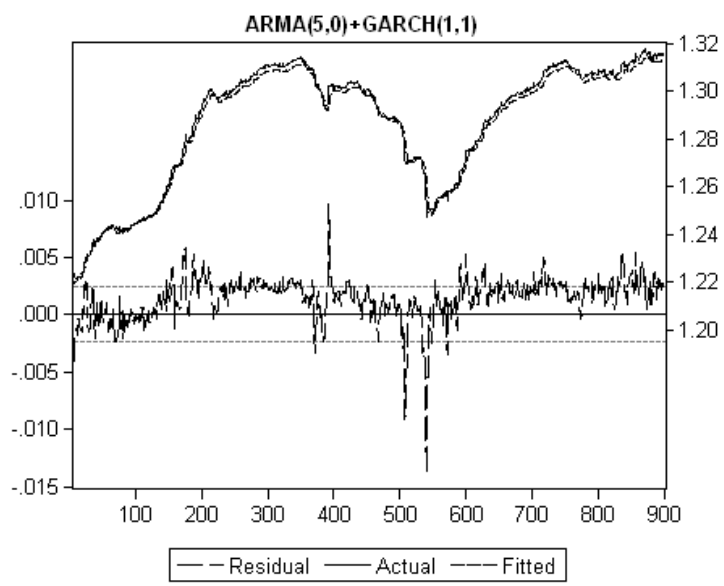

coefficient for leverage effect $\gamma$ from (2) is statistically

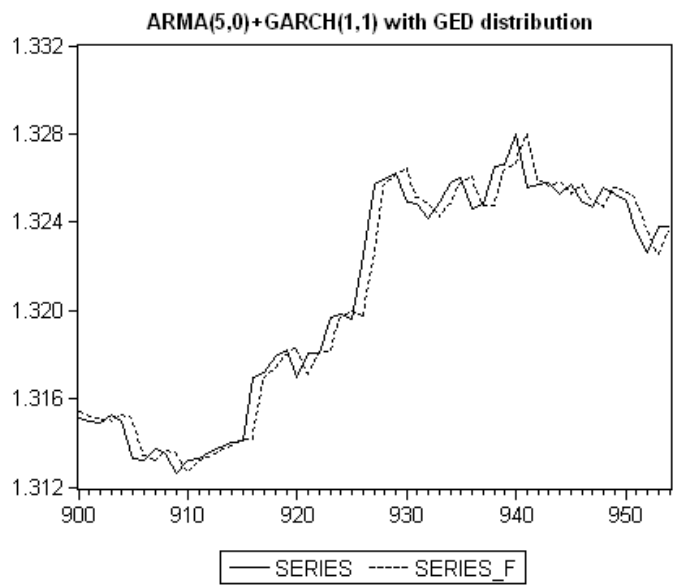

Figure 1 Actual and fitted values of the VUB fund: ARMA(5,0)+GARCH(1,1) model (5) (left). Residuals are at the bottom. Actual time series represents the solid line, the fitted values represents the dotted line. Actual (solid) and forecast (dotted) values of the VUB fund generated from model ARMA(5,0)+GARCH(1,1) with GED error distribution (right).

significant and equals -0.2099535 . This coefficient is negative which means that bad news has larger impact onto volatility (see Figure 2). If we compare the estimated volatility in Figure 2 with the residuals of the VUB fund in Figure 1, we can see that in the period of depression the leverage effects and the bad news cause the asymmetric jump in the volatility.

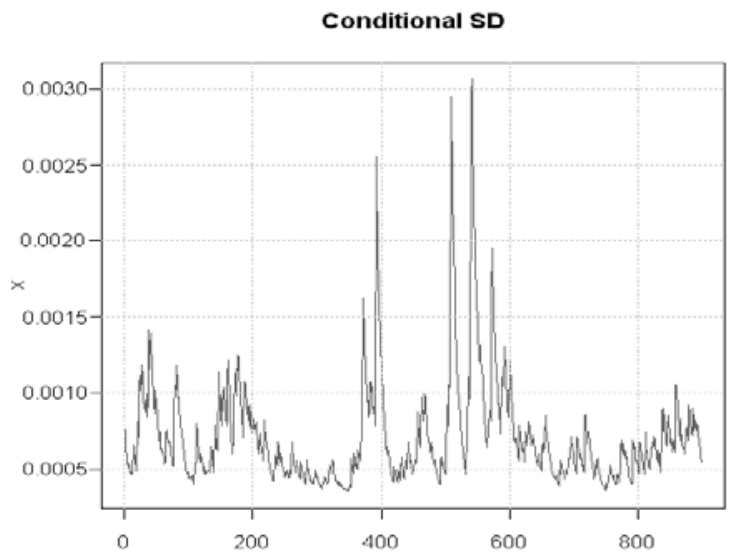

Figure 2 The estimated volatility for $\operatorname{ARMA}(5,0)+$ $\operatorname{ARCH}(1,1)$ process, see (5) and (6).

In many cases, the basic ARCH-GARCH model (1) with Gaussian standard error distribution provides a reasonably good model for analyzing financial time series and estimating conditional volatility. However, there are some aspects of the model which can be improved so that it can better capture the characteristics and dynamics of a particular time series. For this purpose the quantile-quantile (QQ) plots are used. Furthermore, the R system assists in performing residual analysis (computes the Gaussian, Student and generalized residuals with generalized error distribution - GED). The selection of the best model may also be conducted by calculating the values of AIC/BIC criterions. These values are shown in Table 1. The GED error distribution provides the best fit of model (5) because AIC and BIC criterions are the smallest.

As we mentioned above, the estimation of EGARCH model shows the presence of leverage effects. The assumption of normal error distribution is also violated because the alternative error distributions provide better goodness of their fit. These findings indicate the chance of gaining better results in forecasting with using some of these models. Our suspicion was confirmed by computing the statistical summary measure of the model's forecast RMSE. As we can see in Table 2 the smallest RMSE value has just the GARCH model with GED error distribution.

After these findings we can make predictions for the next 54 trading days using the model with the smallest RMSE, i.e. by the $\operatorname{ARMA}(5,0)+$ GARCH $(1,1)$ model with GED error distribution. These predictions are calculated by means software Eviews and shown in Figure 1.

\section{An Alternative Approach}

In this section we show a new approach of function estimation based on granular computing (GC) components for representing neurons in hidden layer of RBF neural network with cloud activation function (CAF). 
The basic components of GC are granules. The granule is defined as a small particle, especially one of numerous particles forming a larger unit. The basic property of a granule is its size. For example, in the cluster analysis, the size of granule can be regarded as the number of elements in the cluster. An important property of granules and granular levels is the granularity. The granularity of a level refers to the collective properties with respect to their size. Granularities are reflected by the size of all granules involved in levels. It enables us to construct a hierarchical structure called a hierarchy. The term hierarchy is used to denote a family of partially ordered granulated levels in which each level consists of a family of interacting granules (see Yao (2008)). The basic function of GC is the granulation. From GC point of view, the process of granulation consists of three basic components: granules, granulated levels and hierarchies. There are two types of the granulation: top-down decomposition of large granules to smaller granules, and the bottom-up combination of smaller granules into larger granules.

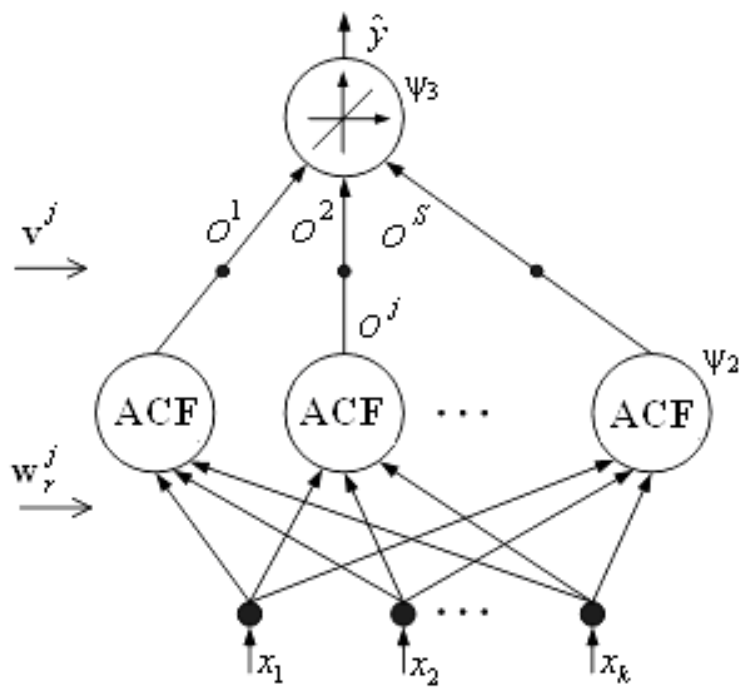

Figure 3 RBF neural network architecture

The structure of a granular neural network is defined by its architecture (see Figure 3). In Figure 3 each circle or node represents the neuron. This neural network consists of an input layer with input vector $\mathbf{x}$ and an output layer with the output value $\hat{y}_{t}$.

The output signals of the hidden layer are

$$
o_{j}=\psi_{2}\left(\left\|\mathbf{x}-\mathbf{w}_{j}\right\|\right),
$$

where $\mathbf{x}$ is a $k$-dimensional neural input vector, $\mathbf{w}_{j}$ represents the hidden layer weights, and $\psi_{2}$ is radial basis (Gaussian) activation function. Note that for an RBF network, the hidden layer weights $\mathbf{w}_{j}$ represent the centers $\mathbf{c}_{j}$ of activation functions $\psi_{2}$.

To find the weights $w_{j}$ or centers of activation functions we used the adaptive (learning) version of K-means clustering algorithm for s clusters (see Marček et al. (2008, 189)). Cluster analysis is regarded as one of the granulation methods, i.e. it say why and how to put objects into the same granule. Granules are extracted from data in the form of clusters, i.e. these entities receive collections of numerical data that exhibit some functional or descriptive commonalities. The final number of clusters provides the number of granules representing RBF neurons in the hidden layer. The centers of clusters are regarded as the means of granules. A family of granules containing every value of input data is called a granulated view. The granulated view of input data consists of a family of overlapping granules (see Figure 4). The above mentioned learning algorithm based on the clustering is regarded as one of the granular methods presenting bottom-up granulation, i.e. input data are combined into larger granules.

The RBF network computes the output data set as

$$
\begin{aligned}
\hat{y}_{t} & =G\left(x_{t}, c, v\right)=\sum_{j=1}^{s} v_{j, t} \psi_{2}\left(x_{t}, c_{j}\right)=\sum_{j=1}^{s} v_{j} o_{j, t}, \\
t & =1,2, \ldots, N,
\end{aligned}
$$

where $N$ is the size of data samples, $s$ denotes the number of the hidden layer neurons (RBF neurons) and $\hat{y}_{t}$ corresponds to the estimated variable $r_{t}$ used in models (4) and (5).

Table 1 AIC, BIC and likelihood function for various types error distribution (model (5) and (6)) made by R system software

\begin{tabular}{|l|c|c|c|}
\hline Model & model.n (Gaussian) & model.t (Student) & model.GED \\
\hline AIC criterion & -10576 & -10778 & -10792 \\
\hline BIC criterion & -10533 & -10730 & -10744 \\
\hline Likelihood function & 5297 & 5399 & 5406 \\
\hline
\end{tabular}

Table 2 Ex post forecast RMSEs for various extensions of GARCH

\begin{tabular}{|l|c|c|c|}
\hline Model distribution & AR(5)+GARCH(1,1) & AR(5)+EGARCH(1,1) & AR(5)+PGARCH(1,1) \\
\hline Gaussian & 0.003461 & 0.001066 & 0.001064 \\
\hline$t$-distribution & 0.002345 & 0.001064 & 0.001063 \\
\hline GED-distribution & 0.001056 & 0.001063 & 0.001062 \\
\hline
\end{tabular}




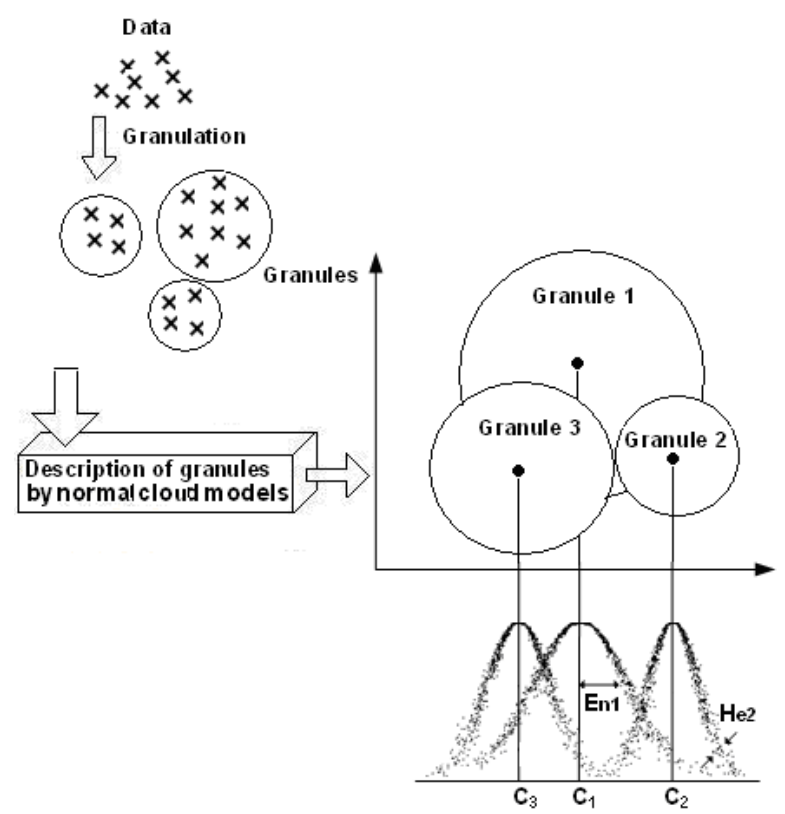

Figure 4 Derivation of granules from input data and their description by normal cloud model.

If the output values $o_{j, t}$ from the hidden layer will be normalized, where the normalization means that the sum of the outputs from the hidden layer is equal to 1 , then the RBF network will compute the "normalized" output data set $\hat{y}_{t}$ as follows

$$
\begin{aligned}
\hat{y}_{t} & =G(x, c, v)=\sum_{j=1}^{s} v_{j t}\left[\psi_{2}\left(x_{t}, c_{j}\right)\right] /\left[\sum_{j=1}^{s} \psi_{2}\left(x_{t}, c_{j}\right)\right], \\
t & =1,2, \ldots, N .
\end{aligned}
$$

The network with one hidden layer and normalized output values $O_{j, t}$ is the fuzzy logic model or the soft RBF network (see Kecman (2001)). In our case, the subjects of learning are the weights $v_{j, t}$ only. These weights can be adapted by the error back-propagation algorithm. The learning rule has the form

$$
\begin{aligned}
& v_{j, t} \leftarrow v_{j, t}+\eta o_{j, t} e_{t}, \\
& j=1,2, \ldots, s, \\
& t=1,2, \ldots, N,
\end{aligned}
$$

where the term $\eta, \eta \in(0,1)$ is a constant called the learning rate, $e_{t}$ is the error given by $e_{t}=y_{t}-\hat{y}_{t}$.

Next, to improve the abstraction ability of soft RBF neural networks with architecture depicted in Figure 3, we replaced the standard Gaussian activation (membership) function of RBF neurons with functions based on the normal cloud concept (see Marček et al. (2008, 212), Marček and Marček (2008)). Then, in the case of soft RBF network, the Gaussian membership function $\psi_{2}(. /$.) in Equation (9) has the form

$$
\begin{aligned}
\psi_{2}\left(x_{t}, c_{j}\right) & =\exp \left[-\left(x_{t}-\mathrm{E}\left(x_{j}\right) / 2\left(E n^{\prime}\right)^{2}\right]\right. \\
& =\exp \left[-\left(x_{t}-c_{j}\right) / 2\left(E n^{\prime}\right)^{2}\right],
\end{aligned}
$$

where $E n^{\prime}$ is a normally distributed random number with mean En (entropy) and standard deviation $\mathrm{He}$ (hyper entropy) which represents the uncertain degree of entropy. $E$ is the expectation operator.

\section{Empirical Comparison}

The RBF NN was trained using the variables and data sets as each ARCH-GARCH model above. In the granular RBF neural network framework, the nonlinear forecasting function $f(\mathbf{x})$ was estimated according to the expressions (9) with RB function $\psi_{2}(. /$.) given by (11). The detailed computational algorithm for the forecast MSE values and the weight update rule for the granular network are shown in Marček and Marček (2008). The results of this application for various architectures of granular RBF networks are shown in Table 3.

Table 3 Ex post forecast RMSEs for various granular RBF NNs (see text for details).

\begin{tabular}{|c|c|c|}
\hline $\begin{array}{c}\text { Number of RBF } \\
\text { neurons }(s)\end{array}$ & $K^{*}$ & RMSE \\
\hline 1 & 1.25 & 0.00719 \\
& 4.0 & 0.00716 \\
\hline 5 & 1.25 & 0.00756 \\
& 4.0 & 0.00758 \\
\hline 10 & 1.25 & 0.00720 \\
& 4.0 & 0.00715 \\
\hline
\end{tabular}

"The value of $K,(K \geq 1)$ is regarded as the rate of overlapping in the distribution of input data. For more details see Marček et al. (2008).

A direct comparison between statistical ARCHGARCH (see Table 2; the RMSE measure for AR(5)+ GARCH $(1,1)$ model with GED distribution is 0.001056). Therefore, the statistical approach is better than the neural network competitor. The achieved ex post accuracy of RBF NN (RMSE $=0.00715$ ), but is still reasonable and acceptable for use in forecasting systems that routinely predict values of variables important in decision making processes. Moreover, as we could see, the RBF NN has such attributes as computational efficiency, simplicity, and ease adjusting to changes in the process being forecast. ARCH-GARCH models require more costs of development, installation and operation in a management system, management comprehension and co-operation, and often a lot of computational time. Another disadvantage of ARCH-GARCH models is that there is not a convenient way to modify or update 
the estimates of the model parameters as each new observation becomes available. One has to periodically completely develop and refit the model. There are no efficient methods for algorithmic estimating the measures of forecasting accuracies (risks) and responsiveness properties. As mentioned in Montgomery et al. (1990) a serious drawback of ARIMA-GARCH models is the investment in time and other resources required to build a satisfactory model. It is doubtful that the improvements in forecast accuracy possible through ARCH-GARCH modeling methodology could justify the cost of the modelbuilding process.

\section{Conclusion}

We have proposed two approaches for determining the forecast accuracy of the forecasting system applied to VUB bond price time series. The first one was based on the latest statistical ARCH-GARCH methodology, the second one on the d granular RBF NN.

At present, there is not a single universally accepted measure of risk in time series forecasting. Because the purpose of forecasting is to reduce the risk in decision making, in our application, we have taken the forecast accuracy (judged by RMSE) as a measure for quantifying the risk.

In direct comparison between statistical ARCHGARCH models and granular RBF NN, the experiment with the daily data indicates that both methodologies yield very little and similar RMSE values. But our experiment shows that RBF NN models are economical and computationally very efficient, well suited for high frequency forecasting. In future research we plan to extend the presented methodologies by applying the exponential smoothing concept to give more weight to recent forecast errors than the older data.

\section{Acknowledgement}

The author thanks Mr. Jan Babel for computational support. The author also wishes to express his gratitude to two anonymous referees, whose valuable remarks helped to improve the quality of the paper.

\section{References}

APPLEBAUM, D. (2004). Lévy Processes and Stochastic Calculus. Cambridge: Cambridge University Press.

http://dx.doi.org/10.1017/CBO9780511755323
BOLLERSLEV, D. (1986). Generalized Autoregressive Conditional Heteroscedasticity. Journal of Econometrics 31: 307327. http://dx.doi.org/10.1016/0304-4076(86)90063-1 BROCK, W., DECHERT, W., SCHEINKMAN, J. (1987). University of Wisconsin at Madison, Department of Economics, Working Paper.

DING, Z., GRANGER, C.W., ENGLE, R.F. (1993). A Long Memory Property of Stock Market Returns and a New Model. Journal of Empirical Finance 1: 83106. http://dx.doi.org/10.1016/0927-5398(93)90006-D

ENGLE, R.F. (1982). Autoregressive Conditional Heteroscedasticity with Estimates of the Variance of United Kingdom Inflation. Econometrica 50 (4): 9871007. http://dx.doi.org/10.2307/1912773

HAN, G.C., ZHANG, Y.L. (2001). Energy equation as generalised mechanical systems, Journal of Harbin Engineering University 22 (4): 69-71, (in Chinese).

HAVLICKÝ, J. (2009). Application of advanced approaches for calculation of operational risk within financial institutions. In: Mathematical Methods in Economics, 109-116. Praha: CZU Praha.

HUANG, C.F. (2007). The basic principle of integrated risk assessment, In: Proc. of the First International Conference on Risk Analysis and Crisis Response. Shanghai 1-6.

HUANG, C.F., RUAN, D. (2008). Fuzzy risks and an updating algorithm with new observation, Risk Analysis 28 (3): 681-694.

http://dx.doi.org/10.1111/j.1539-6924.2008.01057.x

KECMAN, V. (2001). Learning and soft computing: support vector machines, neural networks, and fuzzy logic. Massachusetts: The MIT Press.

KOSKO, B. (1992). Neural networks and fuzzy systems: a dynamical system approach to machine Intelligence. New Jersey: Prentice-Hall International.

MARČEK, M., MARČEK, D. (2008). Granular RBF Neural Network Implementation of Fuzzy Systems: Application to Time Series Modelling, Journal of Mult.-Valued Logic \& Soft Computing 14 (3-5): 101114.

MARČEK, M., PANČIKOVÁ, L., MARČEK, D. (2008). Econometrics \& Soft Computing. Zilina: The University Press of Žilina.

MARČEK, M. (2009). Multiple Statistical Data Analysis and Time Series Models in Economics. Opava: The University Press of Opava.

MONTGOMERY, D.C., JOHNSON, L.A., GARDINER, J.S. (1990). Forecasting and Time Series Analysis. New York: McGraw-Hill. 
NELSON, D.B. (1991). Conditional Heteroskedasticity in Asset Returns: a New Approach. Econometrica 59 (2): 347-370.

http://dx.doi.org/10.2307/2938260

TICHÝ, T. (2006). Model Dependency of the Digital Option Replication: Replication under Incomplete Model. Finance a úvěr - Czech Journal of Economics and Finance 56 (7-8): 361-379.

YAO, Y.Y. (2008). Granular Computing: Past, Present and Future, In: Proc. of the IEEE. 26th - 28th August 80-85.
YOSHIDA, Y. (2003). The valuation of European options in uncertain environment. European Journal of Operational Research, 146: 101-114.

ZIVOT, E., WANG, J. (2005). Modeling Financial Time Series with S-PLUS®. New York: Springer.

ZMEŠKAL, Z. (2005). Value at risk methodology of international index portfolio under soft conditions (fuzzy stochastic approach), International Review of Financial Analysis (14): 263-275.

http://dx.doi.org/10.1016/j.irfa.2004.06.011 